\title{
Are Nucleated Cell Counts Useful in the Diagnosis of Infection in Periprosthetic Fracture?
}

\author{
Stephen Preston MD, Lyndsay Somerville PhD, \\ Brent Lanting MD, James Howard MD
}

Published online: 29 January 2015

(C) The Association of Bone and Joint Surgeons( 2015

\begin{abstract}
Background Evaluating for the possibility of prosthetic joint infection in the setting of periprosthetic fracture is important because it determines the course of treatment. However, fracture-related inflammation can make investigations used in the diagnosis of infection less reliable.

Questions/purposes The purpose of our study was to evaluate synovial fluid nucleated cell counts as a diagnostic test for deep prosthetic infection in patients with periprosthetic fractures around hip and knee arthroplasties. Specifically, we wished to determine the test's properties (sensitivity, specificity, positive predictive value [PPV], and negative predictive value $[\mathrm{NPV}]$ ) using threshold levels of nucleated cell counts as they are otherwise used in the diagnosis of periprosthetic infection.

Methods Billing codes were used to identify all cases of revision total hip arthroplasty (THA), revision total knee arthroplasty (TKA), open reduction and internal fixation (ORIF) of the femur, and ORIF of the tibia at our institution between 2005 and 2013. A total of 2537 charts were

The institution of one or more of the authors (BL, JH) has received, during the period, research support funding from Stryker (Kalamazoo, MI, USA), Smith \& Nephew (London, UK), DePuy (Warsaw, IN, USA), and MicroPort Orthopedics (Arlington, TN, USA).

All ICMJE Conflict of Interest Forms for authors and Clinical Orthopaedics and Related Research ${ }^{\circledR}$ editors and board members are on file with the publication and can be viewed on request. Each author certifies that his or her institution approved the human protocol for this investigation, that all investigations were conducted in conformity with ethical principles of research, and that informed consent for participation in the study was obtained.
\end{abstract}

S. Preston ( () , L. Somerville, B. Lanting, J. Howard

London Health Sciences Centre, 339 Windermere Road,

London, ON N6G 2V4, Canada

e-mail: spresto6@gmail.com identified and reviewed to reveal 269 patients with 269 periprosthetic fractures about a THA or TKA $(10.6 \%$ of charts reviewed). Of these, 27 fractures in 27 patients (10\% of the periprosthetic fractures identified) underwent aspiration of their total joint arthroplasty to rule out infection before surgical intervention. The decision to aspirate was made by the treating surgeon based on clinical suspicion of infection from the patient history, physical examination, and radiographic findings. Nucleated cell counts from joint aspirates were recorded for all 27 patients. Synovial fluid culture results were then used to calculate the sensitivity, specificity, PPV, and NPV of an elevated nucleated cell count in the diagnosis of infection.

Results The specificity, sensitivity, PPV, and NPV of an elevated nucleated cell count in the diagnosis of infection were 64\% (95\% confidence interval [CI, 34.94-75.57]), 100\% (95\% CI, 19.29-100), 18\% (95\% CI, 2.37-45.46), and $100 \%$ (95\% CI, 76.66-100), respectively. Eleven of 27 patients $(41 \%)$ with joint aspirates had elevated nucleated cell counts. Only two of the 11 patients (18\%) with elevated nucleated cell counts had positive synovial fluid cultures. None of the patients with normal nucleated cell counts had positive synovial fluid cultures.

Conclusions Although quite common, an elevated nucleated cell count has moderate specificity and poor PPV in the diagnosis of infection in the setting of periprosthetic fracture.

Level of Evidence Level IV, therapeutic study.

\section{Introduction}

Periprosthetic fracture is a devastating complication of total joint arthroplasty (TJA) for both patients and treating 
surgeons. Unfortunately, the number of periprosthetic fractures is steadily increasing in today's TJA population $[2,7,12-15]$. The etiology behind this increase is multifactorial: the number of TJAs performed has increased during the past decade and patients are living longer and are remaining considerably more active than were patients in previous years $[3,20]$. Generally, the treatment of patients with a periprosthetic fracture is relatively straightforward, because the etiology of the injury is clear on history. However, there are certain circumstances in which periprosthetic joint infection (PJI) must be ruled out to properly manage the injuries, including patients who present with a history of PJI or a prodrome of symptoms consistent with infection, patients who present with a mechanism or radiographic findings that are inconsistent with the severity of their injury pattern, or patients with radiographic findings that may suggest infection. In these situations, the presence or absence of infection will influence management strategies and outcomes of operative fracture fixation or revision surgery.

The diagnosis of PJI can be challenging for orthopaedic surgeons. Currently, the most accepted definition of PJI is based on a consensus statement from the Workgroup of the Musculoskeletal Infection Society (MSIS) [19]. The definition takes several factors into account, including physical findings, microbiologic results, and serologic markers of inflammation, to diagnose PJI. Erythrocyte sedimentation rate (ESR) and C-reactive protein (CRP) levels above $30 \mathrm{~mm} /$ hour and $10 \mathrm{mg} / \mathrm{L}$, respectively, have been associated with infection in previous work by Greidanus et al. [11] and are included in the MSIS definition of PJI. Additionally, an elevated synovial nucleated cell count factors into the diagnosis of infection surrounding a prosthesis. Multiple studies have looked at the use of nucleated cell counts in the diagnosis of PJI. Ghanem et al. [9] found an association between cell counts in excess of $1100 \times 10^{6} / \mathrm{L}$ and PJI in the setting of TKA; cell counts in excess of $3000 \times 10^{6} / \mathrm{L}$ seem to indicate infection in THA
[22]. Unfortunately, despite the work of the MSIS, no consensus has been achieved to help clinicians make the diagnosis of PJI in the setting of periprosthetic fracture.

The purpose of our study was to evaluate synovial fluid nucleated cell counts as a diagnostic test for deep prosthetic infection in patients with periprosthetic fractures around hip and knee arthroplasties. Specifically, we wished to determine the test's properties (sensitivity, specificity, positive predictive value [PPV], and negative predictive value [NPV]) using threshold levels of nucleated cell counts as they are otherwise used in the diagnosis of periprosthetic infection.

\section{Patients and Methods}

We performed a retrospective case series study of all patients admitted with the diagnosis of periprosthetic fracture who underwent a preoperative joint aspirate at a tertiary care center specializing in adult hip and knee reconstruction (London Health Sciences Centre, University Campus, Ontario, Canada) between January 1, 2005, and June 30, 2013.

Ethics research board approval was obtained. We performed a review of our institution's billing codes for the patient admission time period to identify 2537 patients having undergone revision TKA, revision THA, or open reduction and internal fixation (ORIF) for the femur or tibia. Electronic chart review was carried out for the 2537 patients to identify 269 cases of periprosthetic fracture in 269 patients (11\% of charts reviewed). Further review of the 269 charts identified 27 patients (10\%) who underwent preoperative synovial fluid aspirate (Fig. 1). The decision to aspirate was made on a case-by-case basis by the treating surgeon and was based on clinical suspicion of PJI before the fracture. Patients who presented with pain before their injury or with prodromal symptoms/physical findings consistent with infection and those for whom radiographic
Fig. 1 Flowchart shows the process for study patient identification and inclusion.
Billing Codes Used to Identify All Cases of Periprosthetic Hip and Knee Fracture at University Hospital (2005-2013)

Review of 2537 Charts Yielded 269 Periprosthetic Fractures

27 Patients Underwent Joint Aspirates Before Surgical Intervention 
findings (substantial bone loss/component loosening) were suggestive of infection underwent joint aspiration before surgical intervention. No standard algorithm was used to determine which patients would undergo aspiration before surgery. None of the 27 patients were taking any form of antibiotic therapy for concomitant infections before undergoing their joint aspirate. Antibiotics were held in all cases until immediately before the skin incision at the time of surgery. Nucleated cell counts from the 27 synovial fluid aspirates were then used to determine whether elevated cell counts $\left(1100 \times 10^{6}\right.$ cells $/ \mathrm{L}$ for knees, $3000 \times 10^{6}$ cells $/ \mathrm{L}$ for hips) were associated with the presence of PJI. The diagnosis of PJI was based on the presence of positive microbiological culture results from synovial fluid aspirates. Intraoperative cultures were used to confirm the results of the synovial fluid cultures obtained before surgery. All synovial nucleated cell counts, fluid cultures, and intraoperative cultures were performed in our hospital core and microbiology laboratories. All data points were collected and contingency tables were used to calculate the sensitivity, specificity, PPV, and NPV of an elevated nucleated cell count for the diagnosis of periprosthetic infection in the setting of fracture.

Demographic data for the study population were collected and summarized showing mean patient age, gender distribution, and mechanism of injury (Table 1). Periprosthetic fractures about total hip prostheses were classified as either acetabular fractures or according to the Vancouver classification system for femoral fractures [6, 14]. All periprosthetic fractures about total knee prostheses were femoral-sided and were classified according to the Lewis and Rorabeck classification system [21] (Table 2).

Data sets were complete for all 27 patients included in our data analysis. Average values for white blood cell count (WBC), ESR, CRP, and nucleated cell count from synovial aspirate were determined (Table 3). Mean clinical followup for the study cohort was 31 months ( \pm 22 months).

Table 1. Patient demographics $(\mathrm{n}=27)$

\begin{tabular}{ll}
\hline Demographic & Number \\
\hline Average age & 76 years \\
Sex & \\
Men & 7 \\
Women & 20 \\
Mechanism of injury & \\
Fall & 14 \\
Osteolysis & 10 \\
Pathologic fracture & 3 \\
\hline
\end{tabular}

\section{Results}

The specificity and sensitivity of an elevated nucleated cell count in the diagnosis of infection in the setting of periprosthetic fracture were $64 \%$ (95\% confidence interval [CI, 34.94-75.57]) and 100\% (95\% CI, 19.29-100), respectively; the PPV and NPV of elevated nucleated cell count were $18 \%$ (95\% CI, 2.37-45.46) and 100\% (95\% CI, 76.66-100), respectively (Table 4). Two patients from the study cohort were diagnosed with PJI on the basis of positive synovial fluid cultures, which were confirmed intraoperatively by positive tissue cultures with corresponding pathogens. Both infected patients had elevated nucleated cell counts. Nine additional patients presented with elevated nucleated cell counts, all of whom had negative synovial fluid and intraoperative culture results. In

Table 2. Classification of periprosthetic fractures in the study

\begin{tabular}{ll}
\hline Classification & Number \\
\hline Hip & 22 \\
Acetabular & 9 \\
Vancouver A (G:L) & $2(2: 0)$ \\
Vancouver B1 & 0 \\
Vancouver B2 & 6 \\
Vancouver B3 & 3 \\
Vancouver C & 1 \\
Acetabular/Vancouver C & 1 \\
Knee (Lewis and Rorabeck) & 5 \\
Type I & 0 \\
Type II & 1 \\
Type III & 4 \\
\hline
\end{tabular}

Table 3. Average values of preoperative WBC, ESR, CRP, and synovial nucleated cell count

\begin{tabular}{ll}
\hline Laboratory test & Value \\
\hline WBC & $8.73 \times 10^{9} / \mathrm{L}$ \\
ESR & $54.52 \mathrm{~mm} / \mathrm{hour} *$ \\
CRP & $62.15 \mathrm{mg} / \mathrm{L}^{*}$ \\
Nucleated cell count & $3999.1 \times 10^{6} / \mathrm{L}^{*}$
\end{tabular}

* Values consistent with periprosthetic infection in the absence of trauma; WBC = white blood cell count; ESR = erythrocyte sedimentation rate; $\mathrm{CRP}=\mathrm{C}$-reactive protein.

Table 4. Classifying patients according to their nucleated cell count and their infection status

\begin{tabular}{llc}
\hline Parameter & Infection present & Infection absent \\
\hline Elevated cell count & 2 & 9 \\
Normal cell count & 0 & 16 \\
\hline
\end{tabular}


Fig. 2 The distribution of patients in our study was according to nucleated cell counts. Units on the $\mathrm{Y}$-axis are nucleated cells $\times 10^{6} / \mathrm{L}$. Patients 1 and 2 represent both patients who were diagnosed with PJI in the setting of periprosthetic fracture. Solid green and blue lines represent diagnostic thresholds for nucleated cell counts in TKA and THA, respectively.

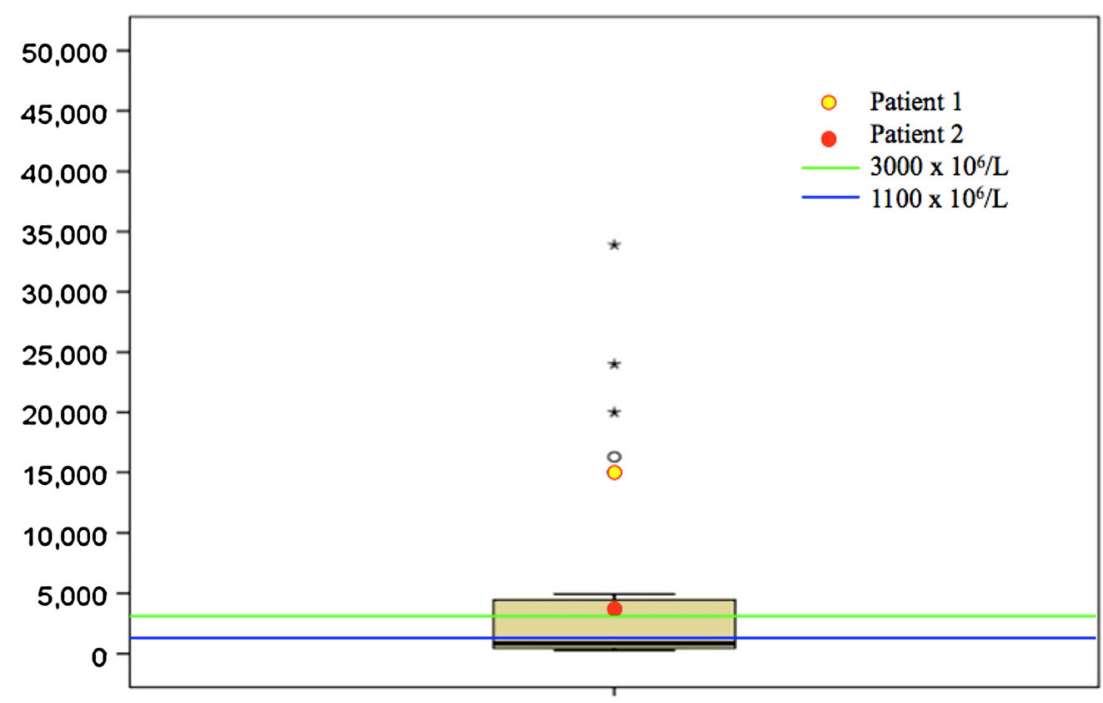

total, 11 of 27 patients (41\%) were found to have elevated nucleated cell counts (Table 4). The distribution of cell counts of all patients as well as previously accepted thresholds for PJI in TKA and THA were compiled and represented graphically (Fig. 2). It should be noted that none of the patients in our study developed a late infection during the remainder of clinical followup.

One of the patients with positive fluid cultures had a periprosthetic acetabular fracture after a fall. The nucleated cell count for this patient was $3953 \times 10^{6}$ cells/L, and their culture grew Escherichia coli. The second patient had a Lewis and Rorabeck Type III supracondylar femur fracture above a TKA, which also occurred after a fall. Synovial aspirate contained $15,000 \times 10^{6}$ nucleated cell/L and fluid culture was positive for Staphylococcus epidermidis.

\section{Discussion}

Periprosthetic fracture is an increasingly common entity facing patients and surgeons after joint arthroplasty. Occasionally, it is necessary to rule out infection as a cause of fracture to properly manage these injuries. Currently, the most widely accepted definition of PJI is that set forth by the MSIS, which uses multiple clinical, microbiological, laboratory, and serologic criteria in unison to diagnose the presence of infection [19]. Unfortunately, thresholds for many of these markers are based on data collected in the absence of periprosthetic fracture. This is an important consideration because commonly used serologic markers such as ESR and CRP have been shown to be less reliable for the diagnosis of PJI in the setting of periprosthetic fracture [4]. The purpose of our study was to evaluate synovial fluid nucleated cell counts (another of the diagnostic criteria set forth by the MSIS [19]) as a diagnostic test for deep prosthetic infection in patients with periprosthetic fractures around hip and knee arthroplasties.

Our study had a number of limitations. First, the retrospective observational design subjects our results to selection bias. The decision to perform aspiration before surgical intervention was made by the treating surgeon on a case-by-case basis and was based on the preoperative clinical suspicion of infection (prodromal symptoms, clinical findings, and/or radiographic findings consistent with possible infection). No standardized criteria were used to determine which patients underwent preoperative aspiration. However, data collection for a prospective study of this nature would not have been feasible given the low prevalence of patients who undergo joint aspiration before surgical management of developing periprosthetic fracture (less than $10 \%$ in our study sample). There are two conceivable ways around this problem, both with their own shortcomings. First, all patients presenting with a periprosthetic fracture could undergo preoperative joint aspiration. However, this would result in subjecting a substantial number of patients to an invasive procedure with questionable use. Furthermore, such a screening-type tool would likely prove cost-prohibitive in a publicly funded healthcare system. The second way would involve the development of more rigorous and standardized criteria for determining which patients should undergo preoperative joint aspiration in the setting of periprosthetic fracture. However, this in itself presents its own set of challenges because markers previously used for the diagnosis of PJI have proved less reliable in the setting of periprosthetic fracture [4] and using clinical features still implies the use of one's own judgment and is thus subject to bias. Another limitation of our study stems from the fact that it did not take into account the possibility of false-negative culture 
results. Although not clearly defined, multiple authors have reported rates of culture-negative periprosthetic infection of the hip and knee ranging from $7 \%$ to $12 \%[1,5,8,10$, 16-18]. The chance that our results were affected by falsenegative culture results is somewhat offset by the fact that all 27 patients had two sets of cultures that were concordant; the aspirate cultures matched the intraoperative culture results for all patients (those who were infected and those who were not). This complication could potentially be avoided if the gold standard for the diagnosis of infection had been the MSIS criteria for PJI [19]. However, many of the criteria listed by the MSIS were not available in the electronic record and, as previously mentioned, serologic markers such as ESR and CRP are less reliable in the setting of periprosthetic fracture. Therefore, we were unable to apply the MSIS criteria for the diagnosis of PJI for the current study. Ideally, we would be able to develop receiver operator curves to identify new nucleated cell count thresholds that could be used for the diagnosis of PJI in the setting of periprosthetic fracture. However, a sample size of 27 with only two events is too small to generate any useful data in this regard. In addition, thresholds for hips and knees would need to be calculated separately, further limiting our sample size.

Our data analysis indicates that an elevated nucleated cell count is only $64 \%$ specific for the diagnosis of PJI in the setting of fracture. Additionally, the PPV of only $18 \%$ implies that less than one-fifth of patients with elevated nucleated cell counts after fracture actually develop PJI. Our results are reinforced by the fact that none of the patients in the study cohort developed late infections during the clinical followup period. Consequently, orthopaedic surgeons should exercise caution when using the nucleated cell count as a diagnostic tool, because it seems to have very limited use when used with currently accepted diagnostic thresholds. Nevertheless, our data indicate that the sensitivity of elevated nucleated cell counts in the diagnosis of PJI in the setting of periprosthetic fracture is $100 \%$. Stated otherwise, if the nucleated cell count is within normal limits, PJI can be ruled out; nucleated cell count could thus serve as an important screening test in patients where clinical suspicion of infection exists. However, the documented false-negative culture rate of $7 \%$ to $12 \%[1,5,8,10,16-18]$ makes it difficult to exclude PJI based on this test alone. The false-negative rate of $0 \%$ in the current study is likely related to the limited sample size.

To our knowledge, our study is the first in the published literature that aims to assess the use of nucleated cell counts for the diagnosis of infection in the setting of periprosthetic fracture. Despite being quite common $(41 \%$ of patients in our study), elevated nucleated cell counts from synovial fluid aspirates have only moderate specificity and poor PPV for the diagnosis of infection in the setting of periprosthetic fracture. Nevertheless, with a sensitivity of $100 \%$, a normal nucleated cell count appears to be an excellent test for ruling out the presence of PJI in the setting of periprosthetic fracture. However, given our small sample size, it would seem that the best approach would be to repeat our basic study with increased patient numbers, perhaps in the context of a multicenter study, to refine diagnostic values for nucleated cell counts used in the diagnosis of infection in the setting of periprosthetic fracture and to use this new information in conjunction with patient history, physical examination, and other laboratory values to properly and safely manage patients with these complex injuries.

\section{References}

1. Berbari EF, Marculescu C, Sia M, Lahr BD, Hanssen AD, Steckelberg JM, Gullerud R, Osmon DR. Culture-negative prosthetic joint infection. Clin Infect Dis. 2007;45:1113-1119.

2. Berry DJ. Epidemiology: hip and knee. Orthop Clin North Am. 1999;30:183-190

3. Bozic KJ, Kurtz SM, Lau E, Ong K, Vail TP, Berry DJ. The epidemiology of revision total hip arthroplasty in the United States. J Bone Joint Surg Am. 2009;91:128-133.

4. Chevillotte CJ, Ali MH, Trousdale RT, Larson DR, Gullerud RE, Berry DJ. Inflammatory laboratory markers in periprosthetic hip fractures. J Arthroplasty. 2009;24:722-727.

5. Duff GP, Lachiewicz PF, Kelley SS. Aspiration of the knee joint before revision arthroplasty. Clin Orthop Relat Res. 1996;331: 132-139.

6. Duncan CP, Masri BA. Fractures of the femur after hip replacement. Instr Course Lect. 1995;44:293-304.

7. Duwelius PJ, Schmidt AH, Kyle RF, Talbott V, Ellis TJ, Butler JB. A prospective, modernized treatment protocol for periprosthetic femur fractures. Orthop Clin North Am. 2004;35:485-492.

8. Font-Vizcarra L, Garcia S, Martinez-Pastor JC, Sierra JM, Soriano A. Blood culture flasks for culturing synovial fluid in prosthetic joint infections. Clin Orthop Relat Res. 2010;468:2238-2243.

9. Ghanem E, Parvizi J, Burnett SJ, Sharkey PF, Keshavarzi N, Aggarwal A, Barrack RL. Cell count and differential of aspirated fluid in the diagnosis of infection at the site of total knee arthroplasty. J Bone Joint Surg Am. 2008;90:1637-1643.

10. Ghanem E, Parvizi J, Clohisy J, Burnett S, Sharkey PF, Barrack R. Preoperative antibiotics should not be withheld in proven cases of periprosthetic infection. Clin Orthop Relat Res. 2007;461:4447.

11. Greidanus NV, Masri BA, Garbuz DS, Wilson SD, McAlinden $\mathrm{GM}, \mathrm{Xu} \mathrm{M}$, Duncan CP. Use of erythrocyte sedimentation rate and C-reactive protein level to diagnose infection before revision total knee arthroplasty. J Bone Joint Surg Am. 2007;89:14091416.

12. Lindahl H. Epidemiology of periprosthetic femur fracture around a total hip arthroplasty. Injury. 2007;38:651-654.

13. Lindahl H, Garellick G, Regner H, Herberts P, Malchau H. Three hundred and twenty-one periprosthetic femoral fractures. $J$ Bone Joint Surg Am. 2006;88:1215-1222.

14. Masri BA, Meek RM, Duncan CP. Periprosthetic fractures: evaluation and treatment. Clin Orthop Relat Res. 2004;420:80 95.

15. McGraw P, Kumar A. Periprosthetic fractures of the femur after total knee arthroplasty. J Orthop Traumatol. 2010;11:135-141. 
16. Pandey R, Berendt AR, Athanasou NA; The OSIRIS Collaborative Study Group; Oxford Skeletal Infection Research and Intervention Service. Histological and microbiological findings in non-infected and infected revision arthroplasty tissues. Arch Orthop Trauma Surg. 2000;120:570-574.

17. Parvizi J, Erkocak OF, Della Valle CJ. Culture-negative periprosthetic joint infection. J Bone Joint Surg Am. 2014;96:430-436.

18. Parvizi J, Ghanem E, Menashe S, Barrack RL, Bauer TW. Periprosthetic infection: what are the diagnostic challenges? J Bone Joint Surg Am. 2006;88(Suppl 4):138-147.

19. Parvizi J, Zimistowski BS, Berbari EF, Bauer TW, Springer BD, Della Valle CJ, Garvin KL, Mont MA, Wongworawat MD, Zalavras CG. New definition for periprosthetic infection-from the
Workgroup of the Musculoskeletal Infection Society. Clin Orthop Relat Res. 2011;469:2992-2994.

20. Pike J, Davidson D, Garbuz D, Duncan CP, O'Brien PJ, Masri BA. Principles of treatment for periprosthetic femoral shaft fractures around well-fixed total hip arthroplasty. J Am Acad Orthop Surg. 2009; 17:677-688.

21. Rorabeck CH, Taylor JW. Classification of periprosthetic fractures complicating total knee arthroplasty. Orthop Clin North Am. 1999;30:209-214.

22. Schinsky MF, Della Valle CJ, Sporer SM, Paprosky WG. Perioperative testing for joint infection in patients undergoing revision total hip arthroplasty. $J$ Bone Joint Surg Am. 2008;90:1869-1875. 